Reprod. Nutr. Dévelop., 1988, 28 (2 B), 499-513.

\title{
Pharmacological manipulation of the mammalian circadian clock : implications for the control of seasonal reproductive cycles
}

F. W. TUREK

Department of Neurobiology and Physiology, Northwestern University, Evanston, Illinois, 60201 USA.

Summary. Although artificial control of the light cycle can be used to regulate the seasonal reproductive cycle of many animals under laboratory conditions, such regulation is often not possible in a standard agricultural environment. An alternative strategy for regulating the seasonal reproductive cycle of photoperiodic animals is to use drugs that either mimic the effects of light on reproductive function or that induce an alteration in the way the circadian clock system is entrained by the light cycle. The ability to use drugs to achieve these objectives has been demonstrated in the laboratory, but it remains to be determined if such an approach can be used to regulate the breeding season of farm animals under normal agricultural conditions.

\section{Introduction.}

Manipulating the reproductive cycle of photoperiodic species in the laboratory is as easy as turning on and off a light switch at the desired time of day. Indeed, even in those photoperiodic species in which the breeding season in nature is confined to only a very limited time of year, reproductive activity in the laboratory can be initiated in any month by careful control of the light cycle prior to and during the induction of reproductive function. However, what is easy in the laboratory is often not possible or practical outside of controlled laboratory conditions. For example, while it is possible to induce estrous cyclicity much earlier in the year in a few female sheep housed in an indoor light-controlled barn by manipulating the light-dark cycle, it is not practical to alter the photoperiod to which 10,000 sheep living outdoors in a pasture in France are exposed.

While stimulation of neuroendocrine-gonadal activity in some animals occurs in response to exposure to the long days of spring and summer, for other animals the short days of autumn and winter are stimulatory to reproductive function (Follett and Follett, 1981). Any attempt to alter the breeding season of a large number of animals under agricultural conditions by either increasing or decreasing 
the amount of light faces many formidable obstacles. Increasing daylength for animals housed outdoors would require extensive and expensive lighting systems that would no doubt be totally impractical for many landscapes. The alternative approach, of housing animals indoors and exposing them to light for select periods of time also puts severe constraints on the ability of farmers to manipulate the reproductive season of herds that can not be easily moved into indoor barns each evening. Trying to decrease the number of hours to which agricultural animals are exposed is an even more difficult task. In view of the fact that exposure to only a few seconds or minutes of relatively low intensity light during the night can induce a long-day reproductive response in animals otherwise held on short days (Earnest and Turek, 1983a ; Ellis and Follett, 1983), light-tight barns would have to be built in order to insure that the animals were indeed exposed to short days despite the presense of long days on the outside. Knowing how difficult it is to control the lighting of small animals housed in animal chambers under well-controlled laboratory conditions, I do not think it would even be possible to prevent any external light from the outdoors from reaching large numbers of animals housed under agricultural conditions.

An alternative approach to the use of light for manipulating the reproductive season of photoperiodic species involves the use of pharmacological agents that would fool the animal into " thinking " it was exposed to long days when in fact the animal was exposed to short days (or vice versa). The key to such a pharmacological approach is the fact that in many mammals and birds photoperiodic time measurement does not involve measuring the absolute length of the day (or night), but instead is dependent upon the phase-relationship between light and the animal's circadian or 24-hr clock (Elliott, 1976 ; Follett and Follett, 1981 ; Underwood et al., 1985 ; Darrow and Goldman, 1986 ; Turek and Van Cauter, 1987). Thus, if light is present at certain times of the circadian cycle, the day will be interpreted as being a long one, regardless of the absolute amount of light to which the animal may be exposed, and the reproductive response of the animal will be the same as if the animals were exposed to normal long days. This relationship between the circadian and reproductive systems in photoperiodic animals suggests two possible strategies for using drugs to alter the photoreproductive response. First, it might be possible to use drugs that stimulate neural pathways carrying light information to or within the photoperiodic timemeasuring system. Such drugs would, in effect, be mimicking light. Second, it might be possible to use drugs to alter the circadian clock system in such a manner that the animal actually " mis-reads " how long the day actually is. Thus, what is normally interpreted as a short day may be read by the circadian/ reproductive system as a long day resulting in the induction of a long-day reproductive response.

As described below, recent experiments in hamsters indicate that it is indeed possible to use specific pharmacological agents to 1) mimic the effects of light on the neuroendocrine-gonadal axis and 2) manipulate the circadian clock in such a manner that the phase relationship between the light cycle and circadian rhythms is altered in such a manner that could lead to an altered photoperiodic response. 


\section{Mimicking effects of light on reproductive system with carbachol.}

In our initial attempts to use drugs to mimic the effects of light on the reproductive system, we used male golden hamsters (Mesocricetus auratus) because it had already been established that even though this species is a longday breeder, maintenance of gonadal function does not require prolonged exposure to light each day. For example, while testicular regression occurs in hamsters exposed to non-stimulatory short days (e.g. LD $6: 18$ or $10: 14)$, it can be prevented by exposing them to as little as 1 second of light near the middle of the night either every day or every other day even though the animals are otherwise maintained on short days (Earnest and Turek, 1983a, 1984). In attempting to find a pharmacological agent that could mimic the effects of light on the reproductive system, we focused our attention on the cholinergic agonist, carbachol, because carbachol can mimic the phase-shifting effects of light on the circadian rhythm of wheel-running activity in mice and hamsters as well as the rhythm of pineal $\mathrm{N}$-acetyltransferase activity in rats (Zatz, 1979; Zatz and Herkenham, 1981 ; Earnest and Turek, 1985). Using two different experimental paradigms, we have demonstrated that in hamsters exposed to either short days (night-interruption paradigm) or constant darkness (T-experiment paradigm), the administration of carbachol at specific circadian times each day can mimic the effects of a short pulse of light delivered at that circadian time and prevent the testicular regression that is observed in control animals.

1. Use of carbachol in night-interruption paradigm (Earnest and Turek, 1983b). - Adult male golden hamsters, housed in individual cages equipped with a running wheel where the circadian rhythm of locomotor activity could be recorded, were transferred from a stimulatory light-dark cycle of LD 14:10 to a nonstimulatory LD 6 : 18 cycle. Upon transfer to short days, the animals were randomly divided into three groups and received intraventricular injections $(2 \mu \mathrm{l})$ of either saline or carbachol $(0.01 \mathrm{M})$ through a cannula that had been stereotaxically placed into the right lateral ventricle during exposure to LD $14: 10$. Group 1 animals received saline injections 8 to 8.5 hours after lights-off, group 2 animals received carbachol 8 to 8.5 hours after lights off while groupe 3 animals were injected with carbachol 1 1.5 hours after lights-on. After 10 weeks the animals were anesthetized and the testes were removed and weighed.

Complete testicular regression was observed in all animals injected with saline during the night as well as in animals injected with carbachol during the light phase of the LD 6 : 18 cycle (fig. 1). In contrast, in 5/9 animals injected with carbachol during the night the inhibitory effects of short days on testicular regression were either totally or partially blocked, while complete testicular regression was observed in the remaining four animals in this group (fig. 1). Importantly, there was a striking correlation between the effects of carbachol on the onset of activity and its effects on testicular weight. Hamsters maintained on an LD 6 : 18 cycle normally begin their activity about 5-7 hours after lights-off, 
and this was the case for all animals injected with saline 8 hours after lights-off. This same phase relationship between activity onset and the timing of the injection was observed in all four animals in which night-time injections of carbachol failed to prevent testicular regression. However, in all five animals with testes weighing more than $1,000 \mathrm{mg}$, the onset of activity occurred after the injection of carbachol; this is the same phase relationship that is established between activity onset and a 1-sec pulse of light when the light pulse is presented $8 \mathrm{hrs}$ after lights-off to animals otherwise maintained on LD $6: 18$ (Earnest and Turek, 1983a). Such a 1-sec pulse of light also blocks short-day induced testicular regression.

FIG. 1. - Mean paired testis weight (bars) for three groups of hamsters exposed to LD 6: 18 for 10 weeks. Group 1 was injected with saline 8 to $8.5 \mathrm{hrs}$ after lightsoff (D) and groups $2 \& 3$ with carbachol, either 8 to 8.5 hrs after lights-off (D) or 1 to $1.5 \mathrm{hrs}$ after lights-on ( $L)$, respectively. Closed circles depict values for individual animals. In animals treated with carbachol during the night, there was a close relationship between the effects of carbachol on the testes and its effects on the circadian rhythm of locomotor activity (see text). From Earnest and Turek (1983b).

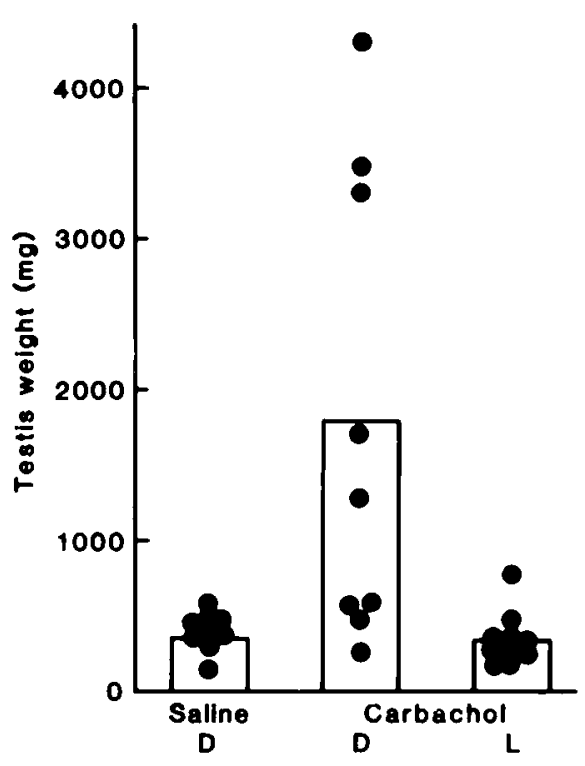

In contrast to nighttime injections, daytime injections of carbachol did not influence short-day induced testicular regression, indicating that carbachol does not have a non-specific effect on the hypothalamic-pituitary-gonadal axis. The observations that nighttime injections of carbachol alter the gonadal response to short days as well as the entrainment of the circadian rhythm of activity, indicates that carbachol may be acting on the circadian system involved in the photic control of reproduction and the rhythm of activity. Since the effects of carbachol injected eight hours after lights-off had an effect on both activity onset and testicular function that was similar to that of a 1-sec pulse of light at this time, these results suggest that injections of carbachol are mimicking the effects of light on the circadian and reproductive systems.

The results of a number of experiments indicate that light induces a photoperiodic response only when it is coincident with a particular phase (or set of phase points) of one or more circadian rhythms (Turek and Campbell, 1979 ; 
Follett and Follett, 1981). In the hamster, the sensitive phase to light appears to begin near the onset of activity and to last for 11 to 12 hours (Elliott, 1976). The finding that an injection of carbachol (or a 1-sec pulse of light) eight hours after lights-off can partially or totally prevent testicular regression in hamsters exposed to LD 6 : 18 is subject to two interpretations : cárbachol, in mimicking the effects of light, was injected during the light-sensitive phase; or, carbachol may be inducing a phase shift in the circadian system such that the 6-hr period of light, which occurs ten hours after the injection, is now coincident with the later portion of the light sensitive phase. In order to determine if carbachol could stimulate neuroendocrine-gonadal activity in the absence of any light, carbachol was administered on a circadian basis to animals housed in constant darkness in what is known as a "T-experiment " paradigm.

2. Use of carbachol in a T-experiment paradigm (Earnest and Turek, 1985). - T-experiments refer to studies in which the period (T) of a potential entraining agent of the circadian system is varied from around 20 to 28 hours. The advantage of this experimental approach is that the effects of a particular entraining stimulus on the reproductive axis can be probed at discrete phase points throughout the circadian cycle simply by varying the period of the stimulus presentation from its normal value of 24 hours. For example, testicular regression occurs in hamsters exposed to 1 hour of light once every $24 \mathrm{hr}$ (LD $1: 23$ ), but regression is prevented in hamsters exposed to the same duration of light once every $23.33 \mathrm{hr}$ (LD $1: 22: 33$ ), because the animals entrain such that light falls at different phases of the circadian cycle under these two photoperiods (Elliott, 1976). If carbachol can indeed mimic the effects of light on both the circadian and reproductive systems, then we predicted that intraventricular injections of carbachol once every $23.33 \mathrm{hr}$ or once every $24 \mathrm{hr}$ would have differential effects on testicular function in a manner similar to that observed when light is presented at the same frequency.

Adult male hamsters were either implanted with a ventricular cannula ( $n=32$ ) or allowed to continue without surgical intervention ( $n=12$ ) while exposed to LD $14: 10$. All the hamsters were then transferred to constant darkness (DD) and the animals with a cannula were randomly divided into four groups and received $1-\mu l$ injections of either saline or $0.01 \mathrm{M}$ carbachol once every $23.33 \mathrm{hr}$ or once every $24.0 \mathrm{hr}$ ( $\mathrm{n}=8$ /group). Animals without a ventricular cannula were exposed to 1 -hr light pulses either once every $23.33 \mathrm{hr}$ or once every $24 \mathrm{hr}$ ( $\mathrm{n}=6 /$ group). All animals were housed with access to a running wheel. After nine weeks, the animals were sacrificed and the testes were removed and weighed.

Regardless of the timing of the injections, marked testicular regression occurred in all hamsters receiving intraventricular injections of saline for nine weeks (fig. 2). In contrast, the effects of carbachol and light on testicular function clearly depended on the period of the treatment cycles. Carbachol, or light, when delivered once every $23.33 \mathrm{hr}$, had a statistically significant effect in maintaining testicular function ( $p<0.01$ ) relative to animals injected with saline once every $23.33 \mathrm{hr}$. However, treatment with carbachol or 1-hr light pulses on a $24 \mathrm{hr}$ basis 
FIG. 2. - Mean ( \pm SEM) paired testis weights of male hamsters injected for 9 weeks in constant darkness with either saline (Sal) or carbachol (Carb) on a $23.33 \mathrm{hr}$ (left panel) or $24.0 \mathrm{hr}$ (right panel) basis. Two additional groups of hamsters were exposed to 1-hr light pulses every 23.33 or $24.0 \mathrm{hr}$. From Earnest and Turek (1985).
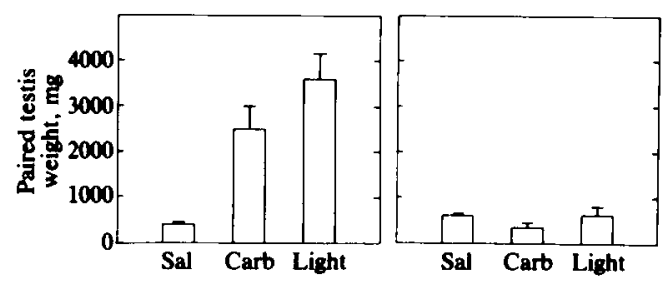

failed to prevent the photoinhibition of testicular function and no significant differences in paired testis weight were observed among groups of hamsters treated with either saline, carbachol or light once every $24 \mathrm{hr}$. Figure 3 depicts the patterns of wheel-running activity from representative animals receiving either saline, carbachol, or $1 \mathrm{hr}$ of light once every $23.33 \mathrm{hr}$. After transfer from LD : $14: 10$ to a $23.33-\mathrm{hr}$ injection cycle, the activity rhythms of all animals receiving saline were observed to free run with periodicities that were $>24 \mathrm{hr}$. No sign of period and/or phase modulation was evident in any of the activity patterns of these animals as different phases of the rhythm passed through the time of saline administration (fig. 3, left). In contrast, injections of carbachol delivered once every $23.33 \mathrm{hr}$ were an effective stimulus for entrainment of the activity rhythm (fig. 3 center). During steady-state entrainment, wheel-running activity was initiated every day 8-9 hr before the time of carbachol administration. Exposure to a 1-hr light pulse once every $23.33 \mathrm{hr}$ also resulted in entrainment of the activity rhythm such that the daily onset of activity preceded lights on by 8 $9 \mathrm{hr}$ (fig. 3 right).
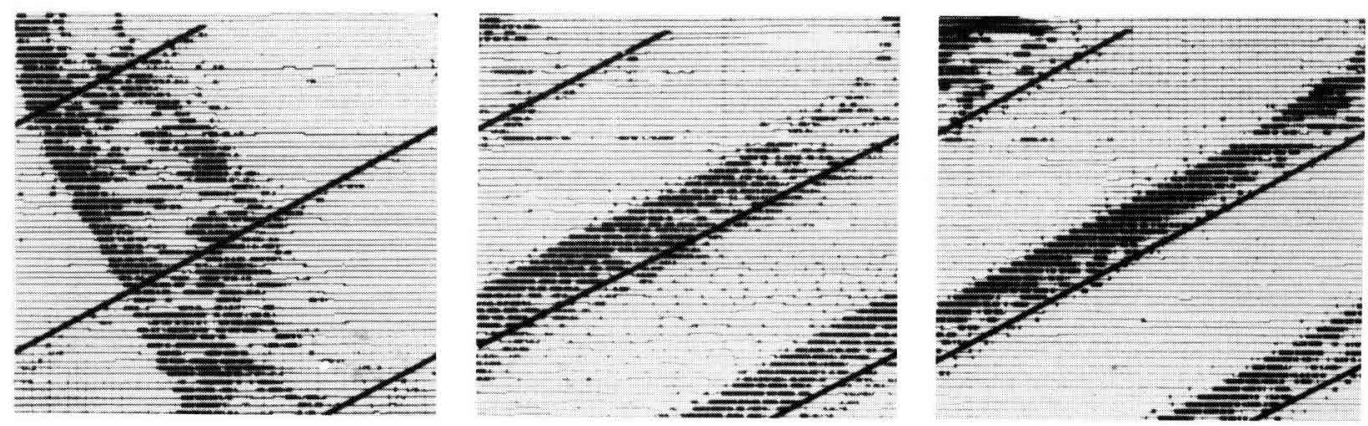

FIG. 3. - Continuous records of whee/-running activity from three representative male hamsters receiving intraventricular injections (Left, saline; center, carbachol) or 1-hr light pulses (right) once every $23.33 \mathrm{hr}$. Diagonal bar in each record represents the time of injection or the onset of the 1-hr light pulse. Successive 24-hr days are plotted from top to bottom. From Earnest and Turek (1985).

These results indicate that intraventricular injections of carbachol can trigger the cascade of biochemical and neural events underlying the effects of light on the circadian and reproductive systems. The administration of carbachol on a circadian basis can entrain the activity rhythm and also mimic the stimulatory 
effects of light on clock-controlled changes in neuroendocrine-gonadal activity in a manner similar to that of 1-hr light pulses presented on a circadian basis.

3. Mechanism of action for effects of carbachol. - The effects of carbachol on circadian and reproductive functions are presumably due to its ability to act as a cholinergic agonist. Thus, it would appear that acetylcholine plays an important role in the mechanism by which light information is transmitted to or within the biological clock involved in the generation of circadian rhythms and photoperiodic time measurement. This hypothesis is supported by the observation that the cholinergic antagonist, mecamylamine, can block the phase-shifting effects of light pulses on the circadian activity rhythm (Keefe et al., 1987). However, at present, the neural site mediating the photomimetic effects of carbachol on circadian rhythms and reproduction has not been defined. Despite the central role played by the eyes in the transfer of photic information to the circadian and reproductive systems, the effects of carbachol are not dependent on the eyes since carbachol-induced phase shifts in circadian rhythms can be induced in blind animals (Zatz and Herkenham, 1981 ; Anderson and Turek, unpublished results). The suprachiasmatic nucleus of the hypothalamus represents a potential locus for the effects of carbachol on the circadian rhythm of activity and reproductive function in the hamster since this structure plays a central role in mediating the effects of light on these processes (Rusak and Zucker, 1979 ; Rusak and Boulos, 1981 ; Moore and Card, 1985 ; Turek, 1985). Further support for the hypothesis that cholinergic neurons in the SCN play a role in mediating the effects of light on the circadian and reproductive systems are the findings that light can alter the firing rate of SCN neurons and can alter acetylcholine concentration within the SCN (Murakami et al., 1984 ; Miller et al., 1987).

4. Difficulty in using the golden hamster to study photomimetic effects of carbachol. - Despite the fact that carbachol can clearly mimic the effects of light on circadian and reproductive functions in the golden hamster under a variety of experimental conditions, we have reached the conclusion that the golden hamster may not be an adequate model for more in-depth studies of the physiological mechanisms by which carbachol acts to alter neuroendocrine-gonadal activity. The main difficulty with this animal model lies in the fact that in order to see clear changes in the photoperiodic reproductive response of male golden hamsters in response to treatment with carbachol $(e . g$. a change in hormone levels or gonadal size), the drug must be delivered over many weeks. Indeed, despite the wealth of information on the general neural and endocrine events associated with the photoperiodic control of reproduction in the golden hamster that has been obtained over the last 15 years, many of the specific neural, endocrine and cellular events which occur in response to a change in day length are not known for this or any other species. The sluggish nature of the photoperiodic response of the golden hamster (as well as in many other species) makes it difficult to determine the key physiological events mediating the effects of light on hypothalamicpituitary-gonadal activity.

Building on earlier studies by Follett, Hoffmann and their colleagues (Hoffmann, 1979, 1982 ; Simpson et al., 1982), we have recently demonstrated 
that dramatic changes in neuroendocrine-gonadal activity can be initiated in the juvenile Djungarian hamster (Phodopus sungorus) very rapidly (within 5-10 days) in response to short (10-min) pulses of light (Milette and Turek, 1986). This rapid response to a brief pulse of light makes the Djungarian hamster an excellent model for probing the neural, cellular and endocrine mechanisms associated with the photoperiodic regulation of seasonal reproductive cycles and in examining the relationship between the circadian and reproductive systems. More detailed analysis of the physiological mechanisms by which carbachol can mimic the effects of light on the reproductive system may require the use of an animal model in which changes in reproductive function occur very rapidly in response to a short pulse of light.

\section{Altering the phase relationship between the light cycle and the circadian system by treatment with triazolam.}

A second strategy for using drugs to alter the photo-reproductive response involves altering the way in which the circadian clock system is entrained to a given light cycle. Circadian rhythms bear a fixed phase relationship to the lightdark cycle such that under entrained conditions, specific phase points of a given circadian rhythm occur at the same phase of the light cycle each day. As the ratio of light to dark is altered, the phase relationship between circadian rhythms and the light cycle can be modified. For example, the onset of activity in male hamsters occurs within 30 min of lights off during entrainment to an LD $14: 10$ cycle, while during entrainment to an LD $6: 18$ cycle, activity starts about five to seven hrs after lights off (Elliott, 1976 ; Ellis and Turek, 1979).

A drug-induced change in the phase relationship between the circadian system and the light cycle could result in a mis-reading of how long the day actually is since different phase points of the circadian clock would be coincident with times of light and dark than under drug-free conditions. Such a change in phase could result in an altered response of the reproductive system to a given daylength. Theoretically, there are at least two possible ways in which a drug could alter the phase relationship between the circadian system and the light cycle. First, the acute administration of a drug might induce a direct phase shift in the circadian clock governing rhythmicity; such a phase shift might alter the entrainment pattern to the entraining light cycle. Second, the chronic administration of a drug might lead to a change in period of the underlying clock ; such a change in period would lead indirectly to a change in the phase relationship between the circadian clock and the light cycle since this phase relationship is dependent on the period of the endogenous clock (Aschoff, 1981 ; Dittendrigh, 1981). Recent experiments indicate that treatment with the short acting benzodiazepine, triazolam, can induce both phase shifts in entrained rhythms as well as induce a change in the period of the underlying circadian clock. These results indicate that triazolam can have both direct and indirect effects in altering the phase relationship between the light cycle and circadian 
rhythms and raise the possibility that such changes in phase could lead to an altered photoperiodic reproductive response.

1. Triazolam-induced phase shifts in the activity rhythm: Direct effects on phase relationship between the light cycle and the circadian activity rhythm. - A single injection of triazolam can induce pronounced phase shifts in the circadian rhythm of locomotor activity in golden hamsters maintained in either constant darkness or constant light (Turek and Losee-Olson, 1986). Importantly, treatment with triazolam can induce both advances as well as delays in the circadian clock ; the direction of the shift depends on the time at which the drug is administered. The observation that a single injection of triazolam can induce a phase shift in the circadian activity rhythm of hamsters maintained under constant lighting conditions raises the possibility that repeated daily injections of triazolam may be capable of altering the entrainment pattern of animals synchronized to a light-dark cycle. Furthermore, since both phase advances and phase delays in the activity rhythm can be induced by varying the time of drug administration, it may be possible to advance or delay the activity rhythm under entrained conditions. To test this hypothesis, the activity rhythm of golden hamsters entrained to an LD 6 : 18 cycle was monitored before, during and after triazolam was injected daily at various times over a 10 to 12 day period (Turek and Losee-Olson, 1988).

In one study, hamsters were maintained undisturbed on an LD 6:18 cycle for at least 33 days before being injected with either $1.25 \mathrm{mg}$ triazolam or vehicle five to six hours before the expected onset of locomotor activity. The injections continued for 11 or 12 days, with the injection each day occurring at the same external clock time as the first injection. After the series of injections, the animals remained on the LD 6:18 cycle, and locomotor activity was recorded for an additional 38 to 72 days. Sixteen animals were injected daily with triazolam and six animals were injected daily with vehicle.

In a second study, 24 hamsters were injected daily with $2.5 \mathrm{mg}$ triazolam for 10 days beginning eight days after transfer to the LD $6: 18$ cycle. The injections were timed to occur three to four hours before $(N=12)$ or $11 \mathrm{hrs}$ after $(N=12)$ the onset of activity on the first day of the injection. After the series of injections, the animals remained on the LD $6: 18$ cycle, and activity was recorded for ten more days. Additional animals maintained on LD $6: 18$ were injected with vehicle for ten days either three to four hours before $(N=6)$ or 11 hours after $(N=6)$ the onset of activity.

Prior to the daily injections, all of the hamsters in the first study showed stable entrainment to the LD 6:18 cycle such that the onset of locomotor activity occurred five to seven hours (mean \pm SEM $=367 \pm 10 \mathrm{~min}$ ) after the offset of the light (fig. 4). Daily injections of vehicle five to six hours before the onset of activity for 11 to 12 days did not consistently alter the phase relationship between the onset of activity and the light-dark cycle (fig. 4-A). The mean phase shift induced by daily injections of triazolam five to six hours prior to the onset of activity was significantly greater $(p<0.05)$ than that observed in animals injected with vehicle at the same time. Indeed, the onset of activity was advanced by at least four hours in six of the 16 triazolam-injected animals (fig. 4-B and 4-C). Interestingly, the original phase relationship between the activity rhythm and the 
light-dark cycle was not reestablished by the end of the study in any of these six animals. The mean onset of activity at the end of the study for these six animals was occurring $181 \pm 28$ min earlier than prior to the injections of triazolam, even though the animals had been maintained under the LD $6: 18$ cycle for 38 to 72 days after treatment with triazolam had been discontinued.
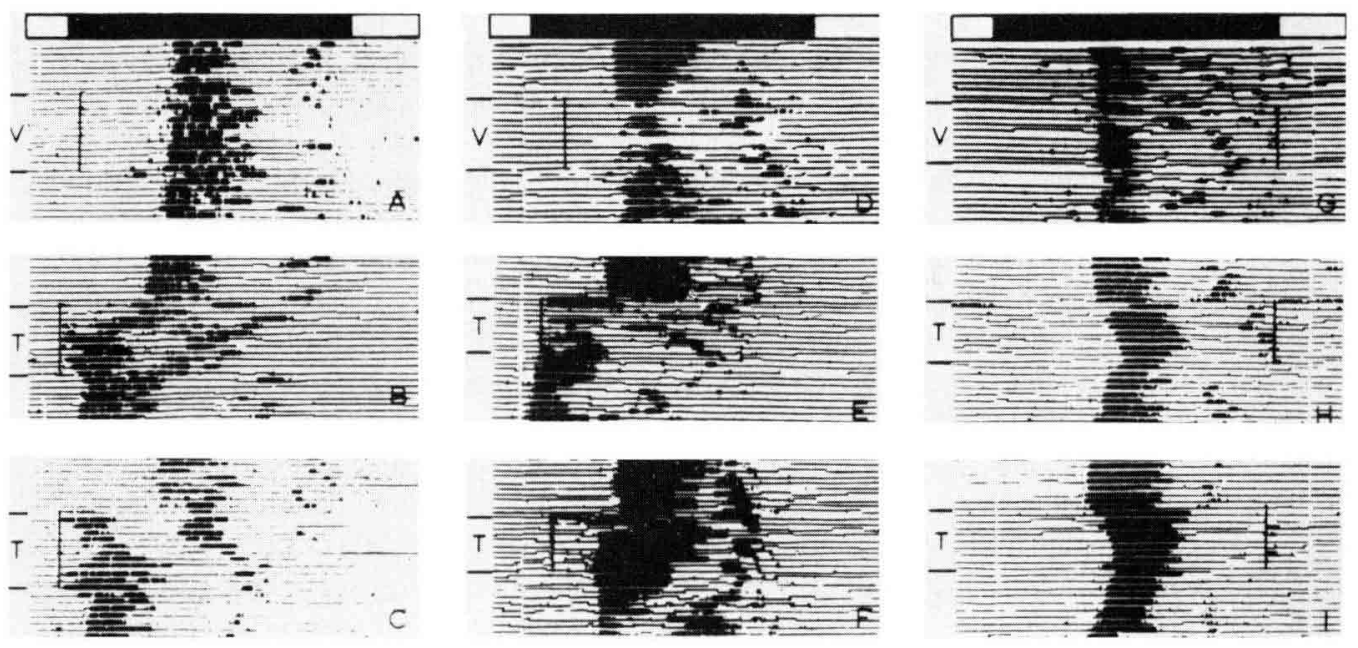

FIG. 4. - Representative sections from the activity records of nine hamsters that were maintained throughout the period shown on the $\angle D$ 6:18 cycle diagrammed at the top of each row of records. Each line represents a single $24 \mathrm{hr}$ time interval. Over a $10-12$ day period near the middle of each record, the animals were injected with either vehicle (V) or triazolam (T) at the same real time each day. The precise time of the injection is designated by the vertical black line on each record. The injections were timed to occur $5-6 \mathrm{hr}$ before (A-C), 3-4 hr before (D-F) or $11 \mathrm{hr}$ after $(\mathrm{G}-\mathrm{I})$ the onset of activity on the day preceding the first injection. From Turek and Losee-Olson (1988).

Prior to the daily injections, all the hamsters in the second study showed stable entrainment to the LD $6: 18$ cycle such that locomotor activity again started five to seven hours (mean \pm SEM $=377 \pm 6 \mathrm{~min}$ ) after the offset of the light (fig. 4). Ten daily injections of vehicle three to four hours prior to, or 11 hours after, activity onset had no consistent effect in altering the phase relationship between the onset of activity and the light-dark cycle (fig. 4-D and 4-G). In contrast, daily injections of triazolam three to four hours prior to, or 11 hours after, activity onset induced consistent phase advances or phase delays, respectively, in the onset of activity. The mean phase shifts in both of these groups were significantly greater $(p<0.05)$ than in the animals injected with vehicle at the same times.

Ten days after the termination of the triazolam injections, the original phase relationship between the activity rhythm and the LD 6:18 cycle was not reestablished in 11 of the 12 animals injected with triazolam three to four hours prior to the onset of activity (fig. 4-E and 4-F). Indeed, compared to the onset of 
activity prior to the triazolam injections, the mean onset of activity for these animals was occurring $125 \pm 27$ min earlier on the last day of LD $6: 18$ (i.e. 10 days after the last injection of triazolam). In contrast, in all 12 animals injected with triazolam 11 hours after activity onset, discontinuation of triazolam treatment resulted in a series of advancing transients such that, by the end of the study, the onset of activity was occurring at about the same time as it was prior to the triazolam injections (fig. 4-H and 4-I).

These results demonstrate that daily injections of triazolam can alter the phase relationship between the entrained circadian rhythm of activity and the light-dark cycle in the hamster. Both advances and delays in the rhythm could be induced, with the direction of the shift being dependent on the time at which triazolam was administered. The times chosen for administering triazolam were based on the phase-shifting effects of a single injection of triazolam on the freerunning activity rhythm under conditions of either constant light or constant darkness (Turek and Losee-Olson, 1986). Injection of triazolam three to six hours before the onset of activity in free-running animals induces an advance in the onset of activity. Similarly, repeated daily injections of triazolam at this same time relative to activity onset in animals entrained to an LD $6: 18$ cycle also induce an advance in the activity rhythm. Conversely, injection of triazolam nine to 12 hours after the onset of activity in free-running animals induces a delay in the activity rhythm. Repeated daily injections of triazolam at this same time relative to activity onset in animals entrained to an LD $6: 18$ cycle also induce a delay in the activity rhythm. Thus the effects of triazolam on the rhythm of locomotor activity are similar under both entrained and free-running conditions.

Following discontinuation of the triazolam injections that induced a delay in the activity rhythm, the onset of activity slowly advanced so that, within 10 days, the original phase relationship between the activity rhythm and the light dark cycle had been reestablished in most of the animals (fig. 4-H and 4-I). The advancing transients were expected, because the triazolam injections had shifted the activity rhythm in such a fashion that more light was now coincident with the late subjective night, a time when light is known to induce advances in the activity rhythm (Daan and Pittendrigh, 1976 ; Ellis et al., 1982 ; Takahashi et al., 1984). In contrast, the advance in the activity rhythm induced by injections of triazolam three to six hours before activity onset was usually maintained even after discontinuation of the injections (figs. 4-B, 4-C, 4-E and 4-F). It should be noted that this is a very unusual entrainment pattern, because the onset of activity in male hamsters maintained on an LD $6: 18$ cycle normally occurs at least five hours after the offset of light (Elliott, 1976 ; Ellis and Turek, 1979). For a complete discussion of why this unusual phase relationship between lights-off and activity onset may have been maintained even after termination of triazolam treatment, see Turek and Losee-Olson (1988).

2. Triazolam-induced changes in the period of the activity rhythm : Possible indirect effects on phase relationship between the light cycle and the circadian activity rhythm. After discovering that daily injections of triazolam could alter the entrainment pattern of hamsters maintained on an LD $6: 18$ cycle, we sought to determine if daily injections of triazolam in free-running animals could entrain the 
circadian rhythm of locomotor activity in the absence of any lighting information (Turek et al., 1987). In this study, blind hamsters were injected intraperitoneally with $0.1 \mathrm{mg}$ of $\mathrm{Tz}(\mathrm{N}=10)$ or vehicle $(\mathrm{N}=9)$ every day for 49 days ; thereafter, the animals were left undisturbed for an additional 15 days. The timing of the daily injections was such that the first injection occurred 3-6 hrs before the onset of activity and subsequent injections occurred at the same real clock time each day. While injections of vehicle did not induce any clear change in the activity rhythm, the first few injections of $\mathrm{Tz}$ induced a pronounced phase advance of the rhythm in 9 of the 10 animals that averaged $262 \pm 41$ minutes (fig. 5). In these 9 animals, daily injections of $T_{z}$ entrained the activity rhythm for about the first 15 days, and then the activity rhythm of all the animals began to free-run with a period less than $24 \mathrm{hrs}$. During the last 10 days of the Tz injections, the mean period of the activity rhythm for the Tz-treated animals $(23.91 \pm 0.02 \mathrm{hrs}$ ) was significantly less ( $p<0.001$ ) than that observed in vehicle-injected animals $(24.26 \pm 0.03 \mathrm{hrs})$. Importantly, this difference in period persisted throughout the 15-days following termination of the injections. These results indicate that it is possible to induce major changes in the period of the circadian clock with daily injections of triazolam, and these changes persist for at least 15 days even after the termination of drug treatment. This change in the endogenous period of the circadian clock may result in an alteration of the entrainment pattern to any given light cycle, although this hypothesis remains to be tested.

FIG. 5. - Continuous record of wheelrunning activity in a blind male hamster injected for 49 days with triazolam $\left(\mathrm{T}_{2}\right)$ at the time indicated by the verticle black line. While daily injections of triazolam entrained the activity rhythm for the first 2-3 weeks, eventually the rhythm began to free-run with a period less than $24 \mathrm{hrs}$ and this shortened period was observed even after termination of drug treatment. From Van Reeth and Turek (unpublished results).

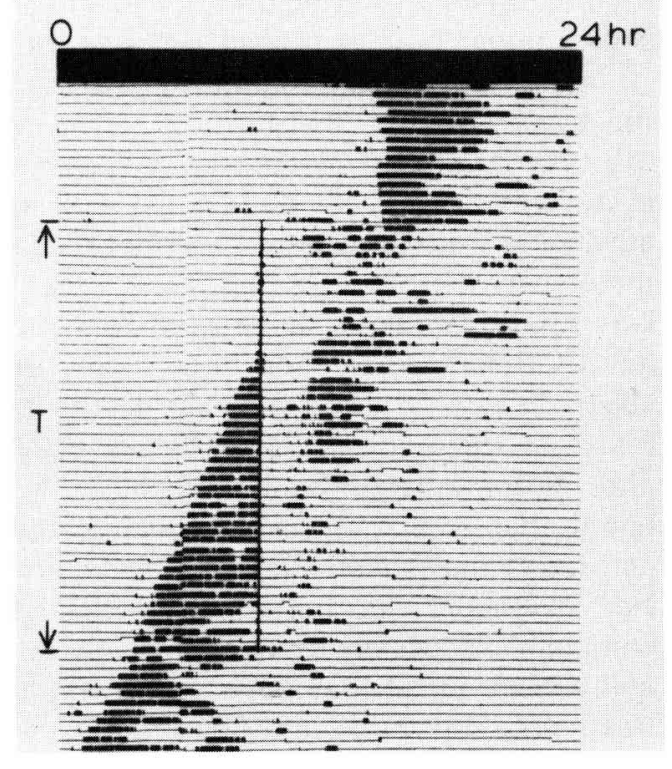

3. Use of benzodiazepines to alter the photoperiodic reproductive response. - Experiments have not yet been carried out to determine if treatment with triazolam can alter the effects of the photoperiod on the neuroendocrine-gonadal 
axis. Therefore, it only remains a theoretical possibility that the photoperiodic response can be altered by either 1) triazolam-induced phase shifts in the circadian clock, and/or 2) triazolam-induced changes in the period of the underlying circadian clock. However, since either manipulation should result in changing the phase relationship between the light-dark cycle and the circadian system, it should be possible to alter the photoperiodic response by appropriate treatment with triazolam. Support for this hypothesis comes from studies performed by Eskes and Zucker (1978) in which they were able to alter the photoperiodic response of male hamsters by treatment with deuterium oxide. While testicular regression normally occurs in hamsters exposed to an LD $10: 14$ light cycle, regression did not occur in animals in which deuterium oxide $(7.5 \%)$ was provided in the drinking water. Deuterium oxide is known to induce a lengthening of the free-running period of the circadian activity rhythm, and under entrained conditions it induces a change in the phase relationship between the light cycle and the activity rhythm. For hamsters maintained on an LD $10: 14$ light cycle and provided with deuterium oxide in the drinking water, activity onset was delayed by about four hours when compared to control animals. This change in phase between activity onset and the light cycle resulted in lights-on occurring about eight hours after the onset of activity; a time when light is known to be stimulatory to neuroendocrine-gonadal activity in this species. It is now of interest to determine if triazolam-induced phase shifts in the way hamsters entrain to short days can also induce a long-day reproductive response.

\section{Conclusion.}

In the beginning of this paper, I suggested that, for a number of reasons, it might not be practical to control the timing of the breeding season of large photoperiodic farm animals under normal agricultural conditions by regulating the light-dark cycle. An alternative approach, would be to use drugs to manipulate the photoperiodic reproductive response, and in this paper two ways in which this could be done are outlined. Drugs could be used to mimic the effects of light on neuroendocrine-gonadal activity, or alternatively, drugs could be used to manipulate the way in which animals entrain to the light cycle such that the phase relationship between the light and the circadian clock is altered in such a manner that leads to an altered photoperiodic response. While I have provided evidence that such pharmacological manipulations may be possible under laboratory conditions, the reader may well ask about the validity of using such an approach under any sort of meaningful agricultural conditions. This is certainly a valid criticism, since none of the pharmacological manipulations of the circadian and reproductive systems detailed above could be used on a large scale in farm animals. However, it should be noted that we are only at the very early stages of our understanding of how drugs can be used to manipulate the photoperiodic response. Many obstacles need to be overcome before one could even design 
field trials. It will be necessary to find drugs that are not only safe but also drugs that can be administered systemically (having to put a cannula into the lateral ventricle would certainly discourage farmers from using carbachol to mimic the effects of light on the reproductive system of their herds). Of primary importance is the development of an inexpensive delivery system in which a chronically implanted capsule could be used to administer the drug at the desired time each day.

Colloquium on a Neuroendocrine mechanisms and light control of reproduction in domestic mammals » I.N.R.A., Nouzilly, 17-18 September 1987.

Acknowledgments. - This work was supported by a National Institutes of Health research grant (HD-09885) and the Upjohn Company.

Résumé. Manipulations pharmacologiques de l'horloge circadienne des Mammifères: applications pour le contrôle des cycles de reproduction.

Bien qu'un cycle lumineux artificiel soit utilisé pour le contrôle du cycle de reproduction chez beaucoup d'animaux en laboratoire, une telle régulation n'est souvent pas possible dans l'environnement agricole ordinaire. Une alternative pour contrôler le cycle saisonnier de reproduction des animaux photopériodiques est l'utilisation de drogues qui, soit miment les effets de la lumière sur la reproduction, soit induisent une modification dans la manière dont l'horloge circadienne est entraînée par le cycle lumineux. La possibilité d'utiliser des drogues pour atteindre ces objectifs a été démontrée dans notre laboratoire, mais il reste à déterminer si une telle approche peut être utilisée pour moduler la saison de reproduction des animaux domestiques dans les conditions normales d'élevage.

\section{References}

ASCHOFF J., 1981. Freerunning and entrained circadian rhythms. In Handbook of behavioral neurobiologv, vol. 4, Biological rhythms, 81-93. Ed. J. ASCHOFF. Plenum Press, New York.

DAAN S., PITTENDRIGH C. S., 1976. A functional analysis of circadian pacemakers in nocturnal rodents. II. The variability of phase response curves. J. comp. Physiol., 106, 253-266.

DARROW J. M., GOLDMAN B. D., 1986. Circadian regulation of pineal melatonin and reproduction in the Djungarian hamster. J. biol. Rhythms, 1, 39-54.

EARNEST D. J., TUREK F. W., 1983a. Effect of one-second light pulses on testicular function and locomotor activity in the golden hamster. Biol. Reprod., 28, 557-565.

EARNEST D. J., TUREK F. W., 1983b. Role for acetylcholine in mediating effects of light on reproduction. Science, 219, 77-79.

EARNEST D. J., TUREK F. W., 1984. Periodic exposure to a brief light signal stimulates neuroendocrine-gonadal activity in golden hamsters. J. Andrology, 5, 64-69.

EARNEST D. J., TUREK F. W., 1985. Neurochemical basis for the photic control of circadian rhythms and seasonal reproductive cycles : role for acetylcholine. Proc. nat. Acad. Sci. USA, 82, 4277-4281.

ELLIOTT J. A., 1976. Circadian rhythms and photoperiodic time measurement in mammals. Fed. Proc., 35, 2339-2346.

ELLIS D. H., FOLLETT B. K., 1983. Gonadotropin secretion and testicular function in golden hamsters exposed to skeleton photoperiods with ultrashort light pulses. Biol. Reprod., 29, 805818. 
ELLIS G. B., TUREK F. W., 1979. Changes in locomotor activity associated with the photoperiodic response of the testes in male golden hamsters. J. comp. Physiol., 132, 277-284.

ELLIS G. B., McKLVEEN R. E., TUREK F. W., 1982. Dark pulses affect the circadian rhythm of activity in hamsters kept in constant light. Amer. J. Physiol., 242, R44-R50.

ESKES G. A., ZUCKER I., 1978. Photoperiodic regulation of the hamster testis : dependence on circadian rhythms. Proc. nat. Acad. Sci., 75, 1034-1038.

FOLLETT B. K., FOLLETT D. E., eds., 1981. Biological clocks in seasonal reproductive cycles, Wright, Bristol.

HOFFMANN K., 1979. Photoperiodic effects in the Djungarian hamster : one minute of light during darktime mimics influence of long photoperiods on testicular recrudescence, body weight and pelage colour. Experientia, 35, 1529-1530

HOFFMANN K., 1982. The effect of brief light pulses on the photoperiodic reaction in the Djungarian hamster Phodopus sungorus. J. comp. Physiol., 148, 529-534.

KEEFE D. L., EARNEST D. J., NELSON D., TAKAHASHI J. S., TUREK F. W., 1987. A cholinergic antagonist, mecamylamine, blocks the phase-shifting effects of light on the circadian rhythm of locomotor activity in the golden hamster. Brain Res., 403, 308-312.

MILETTE J. J., TUREK F. W., 1986. Circadian and photoperiodic effects of brief light pulses in male Djungarian hamsters. Biol. Reprod., 35, 327-335.

MILLER J. D., MURAKAMI D. M., FULLER C. A., 1987. The response of suprachiasmatic neurons of the rat hypothalamus to photic and nicotinic stimuli. J. Neurosci., 7, 978-986.

MOORE R. Y., CARD J. P., 1985. Visual pathways and the entrainment of circadian rhythms. New York Acad. Sci., 453, 123-133.

MURAKAMI N., TAKAHASHI K., KAWASHIMA K., 1984. Effect of light on the acetylcholine concentrations of the suprachiasmatic nucleus in the rat. Brain Res., 311, 358-360.

PITTENDRIGH C. S., 1981. Circadian organization and the photoperiodic phenomena. In Biological clocks in seasonal reproductive cycles, 1-35. Eds. B. K. FOLLETT and D. E. FOLLET. Wright, Bristol.

RUSAK B., BOULOS Z., 1981. Pathways for photic entrainment of mammalian circadian rhythms. Photochem. Photobiol., 34, 267-273.

RUSAK B., ZUCKER I., 1979. Neural regulation of circadian rhythms. Physiol. Rev., 59, 449-526.

SIMPSON S. M., FOLLETT B. K., ELLIS D. H., 1982. Modulation by photoperiod of gonadotrophin secretion in intact and castrated Djungarian hamsters. J. Reprod. Fert., 66, 243-250.

TAKAHASHI J. S., DeCOURSEY P. J., BAUMAN L., MENAKER M., 1984. Spectral sensitivity of a novel photoreceptive system mediating entrainment of mammalian circadian rhythms. Nature, 308, 186-188.

TUREK F. W., 1985. Circadian neural rhythms in mammals. Ann. Rev. Physiol., 47, 49-64.

TUREK F. W., CAMPBELL C. S., 1979. Photoperiodic regulation of neuroendocrine-gonadal activity. Biol. Reprod., 20, 32-50.

TUREK F. W., LOSEE-OLSON S., 1986. A benzodiazepine used in the treatment of insomnia phaseshifts the mammalian circadian clock. Nature, 321, 167-168.

TUREK F. W., LOSEE-OLSON S., 1988. Entrainment of the circadian activity rhythm to the light dark cycle can be altered by a short-acting benzodiazepine, triazolam. J. biol. Rhythms (in press).

TUREK F. W., VAN CAUTER E., 1987. Rhythms in reproduction. In The Physiology of reproduction, 1789-1830. Eds. E. KNOBIL and J. NEILL. Raven Press, New York.

TUREK F. W., VANDERHAEGHEN J. J., VAN REETH O., 1987. Entrainment and the development of tolerance of the mammalian circadian clock to daily injections of triazolam. Neurosci. Abstr., 13, 1040.

UNDERWOOD H., WHITSETT J. M., O'BRIEN T. G., 1985. Photoperiodic time measurement in the male deer mouse, Peromyscus maniculatus. Biol. Reprod., 32, 947-956.

ZATZ M., 1979. Photoentrainment, pharmacology, and phase shifts of the circadian rhythm in the rat pineal. Federation Proc., 38, 2596-2601.

ZATZ M., HERKENHAM M. A., 1981. Intraventricular carbachol mimics the phase-shifting effect of light on the circadian rhythm of wheel-running activity. Brain Res., 212, 234-238. 